Roger Flores Ceccon ${ }^{1}$

Diego Olschowsky Borges ${ }^{2}$

Lucilene Gama Paes ${ }^{3}$

Jonatas Zeni Klafke ${ }^{4}$

Paulo Ricardo Nazário Viecili ${ }^{5}$

${ }^{1}$ Escola de Enfermagem,

Universidade Federal do Rio Grande do Sul. R. São

Manoel, Rio Branco. 90620-

110 Porto Alegre RS. roger.ceccon@hotmail.com

${ }^{2}$ Universidade de Cruz Alta.

${ }^{3}$ Programa de

Pós-graduação em

Enfermagem, Universidade

Federal de Santa Maria.

${ }^{4}$ Faculdade Biomedicina,

Universidade de Cruz Alta.

${ }^{5}$ Curso de Fisioterapia,

Universidade de Cruz Alta.

\section{Mortalidade por doenças circulatórias e evolução da saúde da família no Brasil: um estudo ecológico}

\author{
Mortality due to circulatory disorders and the evolution \\ of family health in Brazil: an ecological study
}

\begin{abstract}
The scope of this study was to analyze deaths due to circulatory disorders in parallel with the evolution of the Family Health Strategy (FHS) in Brazil. It is an ecological and retrospective study based on the temporal evolution of the FHS and mortality rates due to circulatory disorders in Brazil. A description of the inhabitant $\mathbf{x}$ FHS coverage ratio and health indicators related to mortality due to circulatory disorders was carried out. Spearman's rank correlation coefficient test was used for the statistical association. There was a population increase of $15 \%$ in Brazil, evolution of $761 \%$ in the number of FHS units and 5\% increase in mortality due to circulatory disorders. The population $\boldsymbol{x}$ FHS ratio rose from 52,838 (1998) to 7,084 (2006) people assisted by FHS units. North and northeast regions showed growth in mortality rates due to circulatory disorders and in 21 states (81\%) there was a positive correlation between this mortality and FHS units ( $r:>0.7 ; p$ $<0.01)$. Finally, the FHS is regarded as an important public health policy, which has achieved successful results in Brazil since its implementation. However, in general terms, its expansion has not influenced the reduction of mortality due to circulatory disorders, which is an indicator that has increased in the country.
\end{abstract}

Key words Family health, Mortality, Ecological studies
Resumo O objetivo deste estudo foi analisar a mortalidade por doenças circulatórias paralelamente à evolução da Estratégia Saúde da Família no Brasil. Estudo ecológico, retrospectivo, baseado na evolução temporal da ESF e nas taxas de mortalidade por doenças circulatórias no Brasil. Foi realizada uma descrição da razão de cobertura habitante $\boldsymbol{X}$ ESF e dos indicadores de saúde relacionados à mortalidade por doenças circulatórias. Para a associação estatística utilizou-se o teste de Correlação de Spearman. Houve aumento populacional no Brasil em 15\%, evolução de $761 \%$ no número de ESF e 5\% de aumento na mortalidade por doenças circulatórias. A razão população x ESF passou de 52.838 (1998) para 7.084 (2006) pessoas assistidas por ESF. As regiões norte e nordeste apresentaram crescimento nas taxas de mortalidade por doenças circulatórias e em 21 (81\%) estados houve correlação positiva entre mortalidade por doenças circulatórias e ESF $(r:>0,7 ; p<0,01)$. Por fim, considera-se a ESF uma importante política pública de saúde, tendo obtido resultados exitosos no Brasil desde a sua implementação. Entretanto, em um contexto geral, sua expansão não influenciou a redução da mortalidade por doenças circulatórias, tendo apresentado aumento deste indicador no país.

Palavras-chave Saúde da família, Mortalidade, Estudos ecológicos 
Introdução

Na década de 1980, com a implementação do Sistema Único de Saúde (SUS), iniciaram-se as mais importantes mudanças no sistema público de saúde brasileiro, que se apresentava profundamente excludente, baseado em um modelo médico-assistencial privatista, curativo e hospitalocêntrico.

Em 1994, como parte das mudanças propostas, foi instituído o Programa Saúde da Família, hoje denominado Estratégia Saúde da Família (ESF), objetivando reorganizar o modelo assistencial vigente, com caráter substitutivo e complementar das práticas convencionais, com enfoque na atenção preventiva e integral ${ }^{1}$. Propôsse práticas que resultassem melhores condições de saúde para a população, especialmente aquelas em situação de vulnerabilidade social, sem deixar de assistir pessoas com melhores condições de vida ${ }^{2}$. A ESF incorporou os princípios do SUS e despontou como um novo paradigma de atenção à saúde, com pressupostos que criaram uma nova forma de produzir saúde, na perspectiva de conversão do modelo assistencial mecanicista e biomédico.

Entre as ações específicas das ESF destaca-se a atenção integral a pessoas que vivem com doenças crônicas não infecciosas, principalmente doenças circulatórias, que representam a principal causa de morte e incapacidade física no mun$\mathrm{do}^{3}$. Sendo assim, a melhoria das condições socioeconômicas, acesso à serviços de saúde - em especial às ESF - e outras ações específicas podem reduzir o risco de morte . $^{4}$

Embora as taxas de doenças circulatórias mostrem queda em homens e mulheres nos últimos anos ${ }^{5}$, ainda não há associação dessa redução com a evolução das ESF no país ${ }^{6}$, tornando necessárias avaliações sistemáticas da sua expansão, tanto em termos de seu impacto sobre a saúde da população, a prevenção de doenças e a mortalidade, quanto pela avaliação do sistema de saúde como um todo ${ }^{7}$.

Dessa maneira, a expansão da atenção básica, promovida pela implantação da ESF, levounos à formulação da hipótese de que este serviço poderia influenciar na redução temporal da mortalidade por doenças circulatórias, considerando o escopo de suas práticas assistenciais, pautadas na promoção de saúde e prevenção de doenças. Sendo assim, o objetivo deste estudo foi analisar a mortalidade por doenças circulatórias paralelamente à evolução da ESF no Brasil.

\section{Métodos}

Trata-se de um estudo ecológico, retrospectivo, baseado na evolução temporal do número de ESF e nas taxas de mortalidade por doenças circulatórias no Brasil. Segundo Morgenstern ${ }^{8}$, os estudos ecológicos são particularmente relevantes quando se tem por objetivo avaliar intervenções na população, tais como novos programas, políticas ou legislações.

Os dados foram coletados a partir do Sistema de Informação da Atenção Básica, disponíveis no endereço eletrônico do Datasus ${ }^{9}$, do $\mathrm{Mi}$ nistério da Saúde, no período de outubro a dezembro de 2010. Foi utilizada a série temporal entre os anos de 1998 a 2006, não sendo usados os dados a partir de 2007 por não estarem disponíveis no sistema durante a coleta das informações. Os dados sobre mortalidade foram organizados de acordo com a X revisão da Classificação Internacional de Doenças (CID-10) e apresentados por frequência bruta, porcentagem, razão e coeficientes proporcionais a 10.000 habitantes. O número de ESF foi obtido na página do Departamento de Atenção Básica do Ministério da Saúde ${ }^{10}$

Em um primeiro momento, foi realizada uma descrição da razão de cobertura habitante X ESF e dos indicadores de saúde relacionados à mortalidade por doenças circulatórias. Foram utilizados os dados gerais do Brasil e subdivididos pelas regiões sul, sudeste, centro-oeste, norte e nordeste.

A análise estatística foi realizada com o programa SPSS, versão 11.5. A associação entre ESF e mortalidade por doenças circulatórias foi estimada através do teste de Correlação de Spearman, levando em consideração a distribuição não paramétrica dos dados. A matriz de correlação foi construída entre a frequência bruta do número de ESF e do número de mortalidade por doenças circulatórias nos anos de estudo, sendo apresentados os resultados dos 26 estados da federação. Foi definida como variável dependente a mortalidade por doenças circulatórias e como variável independente o número anual de ESF. Foi considerado $\mathrm{p}<0,05$.

A pesquisa respeitou as principais recomendações da última versão da Declaração de Helsinque, sendo o projeto de pesquisa aprovado pelo comitê de ética e pesquisa da Universidade de Cruz Alta (Unicruz). 


\section{Resultados}

A Tabela 1 apresenta os dados relativos à razão de cobertura populacional por ESF e os coeficientes de mortalidade por doenças circulatórias no Brasil, no decorrer dos anos de 1998 a 2006. Na Tabela 2, os mesmos dados estão subdivididos pelas regiões sul, sudeste, centro-oeste, norte e nordeste, na mesma sequência temporal. A Tabela 3 apresenta os valores da Correlação de Spearman entre ESF e mortalidade por doenças circulatórias, de um modo geral no Brasil e distribuídas por estado.

Durante o período analisado houve aumento populacional no Brasil em 15\%, evolução de $761 \%$ no número de ESF e aumento de 5\% na mortalidade por doenças circulatórias. A razão população x ESF passou de 52.838 (1998) para 7.084 (2006) pessoas assistidas, representando aumento na cobertura de $86,5 \%$.

$\mathrm{O}$ crescimento da população foi observado em todas as regiões do país, com predomínio da região centro-oeste, com aumento de $21 \%$. Dessa maneira, no ano de 2006, a região nordeste apresentou a melhor cobertura populacional por ESF, atingido 4.809 pessoas por unidade implementada. Entretanto, no mesmo período, a região sudeste apresentou a cobertura mais elevada, com 9.899 indivíduos por ESF.

Mesmo com a expansão da ESF no país, houve aumento na mortalidade por doenças circulatórias nas regiões norte (33\%), nordeste (39\%) e centro-oeste $(25 \%)$, evidenciando-se pequena redução temporal da mortalidade por doenças circulatórias apenas nas regiões sul (1\%) e su-

Tabela 1. Razão de Cobertura Populacional por ESF e Mortalidade por Doenças Circulatórias por 10.000 habitantes, 1998-2006, Brasil.

\begin{tabular}{cccc}
\hline Ano & População & $\begin{array}{c}\text { População } \\
\text { por ESF }\end{array}$ & $\begin{array}{c}\text { Mortalidade } \\
\text { por doenças } \\
\text { circulatórias }\end{array}$ \\
\hline 1998 & 161.790 .182 & $52.838,07$ & 15,83 \\
1999 & 163.947 .436 & $38.850,10$ & 15,68 \\
2000 & 169.799 .170 & $19.306,33$ & 15,35 \\
2001 & 172.385 .776 & $13.015,16$ & 15,32 \\
2002 & 174.632 .932 & $10.211,85$ & 15,36 \\
2003 & 176.876 .251 & $9.096,70$ & 15,56 \\
2004 & 179.108 .134 & $8.196,42$ & 15,98 \\
2005 & 184.184 .074 & $7.279,72$ & 15,45 \\
2006 & 186.770 .613 & $7.084,30$ & 16,07 \\
\hline
\end{tabular}

ESF: Estratégia Saúde da Família.

Tabela 2. Razão de Cobertura Populacional por ESF e Mortalidade por Doenças Circulatórias por 10.000 habitantes, 1998 - 2006, regiões do Brasil.

\begin{tabular}{cccc}
\hline Ano & População & População & Mortalidade \\
Região & por ESF & $\begin{array}{l}\text { por doenças } \\
\text { circulatórias }\end{array}$
\end{tabular}

Sul

$\begin{array}{llll}1998 & 24.154 .142 & 68.425,33 & 20,68\end{array}$

$1999 \quad 24.445 .843 \quad 54.934,48 \quad 20,03$

$\begin{array}{llll}2000 & 25.107 .616 & 20.396,11 & 19,88\end{array}$

$\begin{array}{llll}2001 & 25.453 .492 & 13.531,89 & 18,95\end{array}$

$\begin{array}{llll}2002 & 25.734 .111 & 10.616,38 & 18,98\end{array}$

$2003 \quad 26.024 .981 \quad 9.422,51 \quad 18,82$

$2004 \quad 26.315 .184 \quad 8.262,22 \quad 19,23$

$\begin{array}{llll}2005 & 26.973 .432 & 7.608,87 & 18,01\end{array}$

$2006 \quad 27.308 .919 \quad 7.078,52 \quad 17,97$

Sudeste

$\begin{array}{llll}1998 & 68.961 .230 & 69.307,77 & 20,07\end{array}$

$\begin{array}{llll}1999 & 69.858 .187 & 59.605,96 & 19,94\end{array}$

$\begin{array}{llll}2000 & 72.412 .411 & 28.475,19 & 19,11\end{array}$

$\begin{array}{llll}2001 & 73.470 .738 & 19.867,70 & 18,77\end{array}$

$2002 \quad 74.447 .443 \quad 14.988,41 \quad 18,57$

$2003 \quad 75.392 .023 \quad 12.815,23 \quad 18,76$

$\begin{array}{llll}2004 & 76.333 .625 & 11.682,53 & 19,11\end{array}$

$\begin{array}{llll}2005 & 78.472 .036 & 10.788,02 & 17,98\end{array}$

$\begin{array}{llll}2006 & 79.561 .023 & 9.899,34 & 18,59\end{array}$

Centro Oeste

$\begin{array}{llll}1998 & 10.994 .817 & 268.166,27 & 10,67\end{array}$

$1999 \quad 11.220 .835 \quad 67.190,63 \quad 10,88$

$\begin{array}{llll}2000 & 11.636 .728 & 19.623,49 & 10,92\end{array}$

$2001 \quad 11.885 .412 \quad 10.583,63 \quad 11,02$

$\begin{array}{llll}2002 & 12.101 .547 & 8.788,34 & 11,55\end{array}$

$\begin{array}{llll}2003 & 12.317 .233 & 8.249,99 & 11,90\end{array}$

$\begin{array}{llll}2004 & 12.532 .306 & 8.090,58 & 12,28\end{array}$

$\begin{array}{llll}2005 & 13.020 .789 & 7.504,78 & 11,52\end{array}$

$\begin{array}{llll}2006 & 13.269 .564 & 7.315,08 & 11,78\end{array}$

Norte

$\begin{array}{llll}1998 & 11.868 .731 & 71.931,70 & 6,64\end{array}$

$\begin{array}{llll}1999 & 12.133 .636 & 29.522,23 & 6,72\end{array}$

$\begin{array}{llll}2000 & 12.900 .704 & 22.752,56 & 6,70\end{array}$

$\begin{array}{llll}2001 & 13.245 .016 & 14.491,26 & 7,11\end{array}$

$\begin{array}{llll}2002 & 13.504 .612 & 11.329,37 & 6,88\end{array}$

$\begin{array}{llll}2003 & 13.784 .895 & 10.777,87 & 7,33\end{array}$

$\begin{array}{llll}2004 & 14.064 .278 & 9.800,89 & 7,44\end{array}$

$\begin{array}{llll}2005 & 14.698 .834 & 8.610,92 & 7,48\end{array}$

$\begin{array}{llll}2006 & 15.022 .071 & 7.803,67 & 7,85\end{array}$

Nordeste

$1998 \quad 45.811 .262 \quad 37.244,93 \quad 10,10$

$\begin{array}{llll}1999 & 46.288 .935 & 25.874,20 & 10,02\end{array}$

$\begin{array}{llll}2000 & 47.741 .711 & 13.605,50 & 10,20\end{array}$

$\begin{array}{llll}2001 & 48.331 .118 & 9.452,59 & 10,85\end{array}$

$\begin{array}{llll}2002 & 48.845 .219 & 7.433,45 & 11,19\end{array}$

$2003 \quad 49.357 .119 \quad 6.622,45 \quad 11,43$

$2004 \quad 49.862 .741 \quad 5.855,87 \quad 12,12$

$\begin{array}{llll}2005 & 51.018 .983 & 4.934,14 & 12,85\end{array}$

$2006 \quad 51.609 .036 \quad 4.809,79 \quad 14,67$

ESF: Estratégia Saúde da Família. 
Tabela 3. Coeficiente de Correlação de Spearman entre o número de ESF e mortalidade por doenças circulatórias nos estados brasileiros - 1998 a 2006.

\begin{tabular}{lc}
\hline Mortalidade por \\
Acre & $0,801^{* *}$ \\
Alagoas & $0,817^{* *}$ \\
Amapá & $-0,423$ \\
Amazonas & $0,921^{* *}$ \\
Bahia & $0,810^{* *}$ \\
Ceará & $0,815^{* *}$ \\
E Santo & $0,899^{* *}$ \\
Goiás & $0,930^{* *}$ \\
Maranhão & $0,976^{* *}$ \\
Mato Grosso & $0,966^{* *}$ \\
Mato Grosso do Sul & $0,932^{* *}$ \\
Minas Gerais & $0,895^{* *}$ \\
Pará & $0,906^{* *}$ \\
Paraíba & $0,945^{* *}$ \\
Paraná & 0,407 \\
Pernambuco & $0,776^{*}$ \\
Piauí & $0,955^{* *}$ \\
Rio de Janeiro & 0,207 \\
Rio Grande do Norte & $0,941^{* *}$ \\
Rio Grande do Sul & $-0,765^{*}$ \\
Rondônia & $0,880^{* *}$ \\
Roraima & $0,860^{* *}$ \\
Santa Catarina & $0,711^{*}$ \\
São Paulo & 0,447 \\
Sergipe & $0,901^{* *}$ \\
Tocantins & $0,964^{* *}$ \\
GERAL & $0,935^{* *}$ \\
\hline ara &
\end{tabular}

${ }^{* *} \mathrm{p}<0,01 ;{ }^{*} \mathrm{p}<0,05$

deste $(6 \%)$, ainda assim com as taxas mais altas do Brasil.

Ao analisar os dados dos 26 estados da federação, pôde-se perceber que em 21 houve correlação positiva entre ESF e mortalidade por doenças circulatórias ( $r>0,7 ; \mathrm{p}<0,05)$, quatro não apresentaram correlação significativa e apenas o estado do Rio Grande do Sul apresentou correlação negativa ( $\mathrm{r}:-0,765, \mathrm{p}<0,01)$. Em um contexto geral, o Brasil apresentou correlação positiva entre ESF e mortalidade por doenças circulatórias (r: 0,935; p <0,01).

\section{Discussão}

Os dados deste estudo demonstraram que, mesmo com o aumento na cobertura populacional pelas ESF no Brasil, não houve redução na mortalidade por doenças circulatórias. Foi possível encontrar diferenças regionais importantes, principalmente entre a região sul, com diminuição temporal nas taxas de mortalidade, e as regiões centro-oeste e norte, onde ocorreu aumento dos indicadores estudados.

A implementação das ESF se deu em um processo lento, sendo que, nos primeiros quatro anos de sua implantação (1994-1998), abrangeu 3,51\% da população brasileira; ao final de 1998, este percentual atingiu 6,57\%. No ano de 1999, existiam 4.114 unidades de ESF distribuídas em 1.646 municípios, representando 30\% dos municípios brasileiros, 14 milhões de pessoas e 8,8\% da população do país. Em setembro de 2002, esses números cresceram significativamente, atingindo 16.463 equipes, 4.161 municípios e 54 milhões de pessoas ${ }^{11}$.

No ano de 2003, o Ministério da Saúde tinha como meta dobrar o número de ESF para os próximos quatro anos, pretendendo atingir 100 milhões de pessoas, cerca de $77 \%$ da população brasileira, implantando, ao final de 2006, 32 mil equipes de $\mathrm{ESF}^{8}$. Os dados deste estudo mostraram que até o ano de 2006 havia 26.364 ESF no Brasil, 17\% menos do esperado.

O processo de implementação das ESF ocorreu diante de um quadro social complexo, onde a pobreza e as desigualdades sociais, atreladas à dificuldade de acesso e à falta de trabalhadores implicados com a mudança podem ter contribuído para o aumento na mortalidade por doenças circulatórias. Muitos problemas foram superados, ainda que persistam dificuldades relevantes e que podem estar influenciando as práticas assistenciais de saúde, em especial aquelas voltadas à prevenção das doenças circulatórias. A Saúde da Família se constituiu como uma política que implica maiores investimentos em recursos humanos e técnicos, o que configura como desafio para sua implantação $0^{5,12}$.

A ESF, enquanto política principal da atenção básica, possui papel fundamental na prevenção, diagnóstico e acompanhamento das doenças circulatórias, devendo prestar uma assistência resolutiva e abrangente, de forma que encaminhamentos e referências ocorram somente em casos necessários, sendo sua responsabilidade o cuidado de forma integral ${ }^{13}$.

Com relação às práticas assistenciais das ESF direcionadas às pessoas que vivem com doenças circulatórias, considera-se necessário repensar as tarefas estabelecidas para o seu funcionamento, voltando-se para ações de promoção à saúde, prevenção e tratamento, em articulação plena entre profissionais, gestores e população, chaman- 
do-os para a participação mútua no planejamento de ações focadas às doenças circulatórias, em um processo de coparticipação ${ }^{14}$.

Dessa forma, as doenças circulatórias apresentam-se como um desafio para as políticas públicas de saúde e, embora alguns estudos apresentem queda nas taxas de morbidade e mortalidade $^{15}$, o mesmo não pôde ser evidenciado neste estudo, que constatou crescimento significativo nestes indicadores. Sendo assim, a mortalidade por doenças circulatórias ainda é alta no Brasil, associando-se muitas vezes com piores condições de vida, vulnerabilidade social, exclusão, falta de acesso a serviços de saúde e pobreza ${ }^{16}$.

Evidenciou-se redução da mortalidade por doenças circulatórias apenas nas regiões sul e sudeste, indo ao encontro de pesquisas que demonstraram diminuição somente em regiões mais desenvolvidas, de forma tardia e discreta ${ }^{17,18}$. Sabe-se que a prevenção primária, a secundária e o controle das doenças circulatórias é mais efetivo em regiões mais desenvolvidas como a Sul e a Sudeste ${ }^{19}$, fato que pode ter sido corroborado com os dados desta pesquisa.

Alguns estudos realizados no sul do Brasil, ao longo de 2007 a 2009, apontaram alta prevalência de indivíduos que não sabiam ser portadores de fatores de risco para doenças circulatórias $^{20,21}$. Este fato encaminha-nos à constatação de que algumas ações passíveis de serem realizadas nas ESF, como educação em saúde, melhor controle dos fatores de risco, diagnóstico precoce e tratamento adequado não estão sendo efetivamente realizados, o que influencia na morbimortalidade das doenças circulatórias.

Para um adequado acompanhamento do comportamento das doenças circulatórias, fazse necessário monitorar o conjunto de séries históricas de mortalidade, e avaliar não somente o comportamento da doença, mas também, de forma indireta, as medidas de prevenção primária e secundária empregadas para seu controle, neste caso referentes à $\mathrm{ESF}^{22}$.

Alguns estudos demonstram eficiência da ESF a partir dos impactos positivos produzidos nos indicadores de saúde e da garantia de maior equidade em saúde no país. Entretanto, constataram que a estratégia está em construção e necessita de qualificação para efetivar a mudança do modelo assistencial e obter resultados mais expressi$\operatorname{vos}^{23,24}$. O que refletiria na diminuição da mortalidade por doenças circulatórias. A maior parte das ações realizadas pela ESF ainda segue o modelo tradicional de assistência, priorizando ações curativas e individualizadas, o que limita seu po- tencial de atuação e influencia nos resultados obtidos ${ }^{25}$.

Entre as limitações deste estudo destaca-se que o Sistema de Informação sobre Mortalidade, do Ministério da Saúde, divulga apenas a causa básica do óbito, o que dificulta a avaliação de outras causas. Outra limitação está associada às subnotificações, que resultam em informações distorcidas do real perfil de mortalidade ${ }^{26}$.

As diferenças encontradas nos valores dos coeficientes analisados não podem ser entendidas exclusivamente como resultantes das intervenções das ESF, pois não foi de alcance do estudo o controle dos fatores de confundimento ${ }^{8,27}$. Sendo assim, os resultados deste estudo permitem apenas aventar hipóteses sobre uma possível associação entre a ESF e os níveis de mortalidade por doenças circulatórias, que deve ser mais bem explorada a partir do uso de outros delineamentos.

A proposta desta pesquisa foi analisar a mortalidade por doenças circulatórias em consonância com a implementação das ESF, principal estratégia de organização da atenção básica, não sendo possível avaliar outros programas ou serviços que influenciaram este indicador. Assim, estes resultados podem também ser atribuídos ao aumento populacional, ao envelhecimento, à dificuldade de acesso e diagnóstico precoce e a outros fatores, podendo inclusive ser escopo de novas pesquisas.

Por fim, considera-se a ESF uma importante política pública de saúde, tendo obtido resultados exitosos no Brasil desde a sua implementação. Entretanto, em um contexto geral, sua expansão não influenciou a redução da mortalidade por doenças circulatórias, tendo apresentado aumento deste indicador no país.

\section{Colaboradores}

RF Ceccon participou das etapas de concepção, delineamento, análise, interpretação dos dados, redação do artigo, revisão crítica e aprovação da versão a ser publicada. DO Borges participou da concepção, delineamento, a análise e interpretação dos dados. LG Paes participou da redação do artigo, da revisão crítica e da versão a ser publicada. JZ Klafke participou da redação do artigo e da revisão crítica. PRN Viecili participou das etapas de concepção, delineamento, análise, interpretação dos dados, redação do artigo, revisão crítica e aprovação da versão a ser publicada. 


\section{Referências}

1. Brasil. Ministério da Saúde (MS). Saúde da Família: Uma estratégia para a reorientação do modelo assistencial. Brasília: MS; 1998.

2. Fernandes LCL, Bertoldi AD, Barros AJD. Utilização dos serviços de saúde pela população coberta pela Estratégia de Saúde da Família. Rev Saude Publica 2009; 43(4):595-603.

3. Organização Panamericana de Saúde (OPAS), Organização Mundial de Saúde (OMS). Doenças crônico-degenerativas e obesidade: Estratégia mundial sobre alimentação saudável, atividade física e saúde. Brasília: OPAS, OMS; 2003.

4. Mansur AP, Souza MFM, Timerman A, Avakian SD, Aldrighi JM, Ramirez JAF. Tendência do risco de morte por doenças circulatórias, cerebrovasculares e isquêmicas do coração em treze Estados do Brasil, de 1980 a 1998. Arq. Bras. Cardiol 2006; 87(5): 641-648.

5. Mansur AP, Timerman A, Souza MFM, Serrano CV, Santos RD. Tendência da Mortalidade por Doenças Circulatórias no Brasil de 1979 a 1996. Arq Bras Cardiol 2001; 76:497-503.

6. Conill EM. Ensaio histórico-conceitual sobre a Atenção Primária à Saúde: desafios para a organização de serviços básicos e da Estratégia Saúde da Família em centros urbanos no Brasil. Cad Saude Publica 2008; 24(Supl. 1):S7-S27.

7. Bodstein R. Processo decisório e avaliação em saúde: ampliando o debate sobre o Programa Saúde da Família. Cien Saude Colet 2009; 14(Supl. 1):13361338.

8. Morgenstern H. Ecologic studies. In: Rothman KJ, Greenland S. Modern epidemiology. Philadelphia: Lippincott Williams \& Wilkins; 1998. p. 459-480.

9. Brasil. Ministério da Sáude (MS). Departamento de Informática do SUS. Sistema de Informação da Atenção Básica. [acessado 2013 mar 26]. Disponível em: www.datasus.gov.br

10. Brasil. Ministério da Saúde (MS). Departamento de Atenção Básica. [acessado 2013 mar 26]. Disponível em: http://dab.saude.gov.br/portaldab

11. Brasil. Programa Saúde da Família: ampliando a cobertura para consolidar a mudança do modelo de Atenção Básica. Rev. Bras. Saude Mater. Infant 2003; 3(1):113-125.

12. Brasil. Ministério da Saúde (MS). Fundação Oswaldo Cruz. Saúde da Família: avaliação da implementação em dez grandes centros urbanos: síntese dos principais resultados. 2a Edição. Brasília: MS; 2005.

13. Alfradique ME, Bonolo PR, Dourado I, LimaCosta MF, Macinko J, Mendonça CS, Oliveira VB, Sampaio LFR, Simoni C, Turci MA. Internações por condições sensíveis à atenção primária: a construção da lista brasileira como ferramenta para medir o desempenho do sistema de saúde (Projeto ICSAP - Brasil). Cad Saude Publica 2009; 25(6):1337-1349.

14. Rosa WAG, Labate RC. Programa Saúde da Família: A construção de um novo modelo de assistência. Rev Latino-am Enfermagem 2005; 13(6):1027-1034.

15. Mansur AP, Lopes AA., Favarato D, Avakian SD, César LAM, Ramires JAF. Transição epidemiológica da mortalidade por doenças circulatórias no Brasil. Arq. Bras. Cardiol 2009; 93(5):506-510.
16. Ishitani LH, Franco GC, Perpétuo IHO, França E. Desigualdade social e mortalidade precoce por doenças cardiovasculares no Brasil. Rev Saude Publica 2006; 40:684-91.

17. Uemura K, Pisa Z. Trends in cardiovascular disease mortality in industrialized contries since 1950. Wld Hlth Statist Quart 1988; 41(3-4):155-178.

18. Thorvaldsen P, Kuulasmaa K, Rajakangas A-M, Rastenyte D, Sarti C, Wilhelmsen L. WHO MONICA Project. Stroke Trends in the WHO MONICA Project. Stroke 1997; 28(3):500-506.

19. Souza MFM, Alencar AP, Malta DC, Moura L, Mansur AP. Análise de Séries Temporais da Mortalidade por Doenças Isquêmicas do Coração e Cerebrovasculares, nas Cinco Regiões do Brasil, no Período de 1981 a 2001. Arq Bras Cardiol 2006; 87(6):735-740.

20. Richter CM, Bündchen DC, Dipp T, Belli KC, Panigas TF, Panigas CF. Unawareness of presence for cardiovascular disease risk factors in a Brazilian regional population. Circulation 2008; 118:139.

21. Richter CM, Bettinelli LA, Pasqualotti A, Borges DO, Daltrozo PRO, Klafke JZ, Viecili PRN. Avaliação do conhecimento e da presença de fatores de risco cardiovascular em idosos de município do sul do Brasil. Rev. Bras. Cardiol 2010; 23(5):277-285.

22. Latorre MRDO, Cardoso MRA. Análise de séries temporais em epidemiologia: uma introdução sobre os aspectos metodológicos. Rev Bras Epidemiol 2001; 4(3):145-152.

23. Andrade LOM, Barreto ICHC, Goya N, Júnior TM. Organização da atenção básica e Estratégia Saúde da Família no município de Sobral - Ceará - Brasil: principais avanços e desafios na perspectiva de produzir mudanças positivas na saúde. In: Brasil. Ministério da Saúde (MS). Experiências e Desafios da Atenção Básica e Saúde Familiar: caso Brasil. Brasília: MS; 2004. p. 143-179.

24. Henrique F, Calvo MCM. Avaliação do Programa Saúde da Família nos municípios do Estado de Santa Catarina, Brasil. Cad Saude Publica 2008; 24(4):809819.

25. Gomes KO, Cotta RMM, Euclydes MP, Targueta CL, Priore SE, Franceschini SCC. Avaliação do impacto do Programa Saúde da Família no perfil epidemiológico da população rural de Airões, município de Paula Cândido (MG), 1992-2003. Cien Saude Colet 2009; 14(Supl. 1):1473-1482.

26. Yuen EJ. Severity of illness and ambulatory caresensitive conditions. Med Care Res Rev 2004; 61(3): 376-391.

27. Medronho RA. Estudos ecológicos. In: Medronho RA, Carvalho DM, Bloch KV, Luiz RR, Werneck GL, organizadores. Epidemiologia. São Paulo: Editora Atheneu; 2005. p. 191-198.

Artigo apresentado em 22/03/2012

Aprovado em 20/04/2012

Versão final apresentada em 17/05/2012 